\title{
Mathematische Paradigmen in der Finanzwirtschaft
}

\author{
Hans Bühlmann
}

Hans Bühlmann wurde 1930 in Chur geboren. Er studierte an der ETH in Zürich und doktorierte dort 1959. Nach einigen Jahren als Mathematiker bei einer Versicherungsgesellschaft wurde er 1966 als Professor für Mathematik an die ETH berufen, wo er vor allem die Gebiete der Wahrscheinlichkeitsrechnung, der Statistik und der Versicherungsmathematik betreute. Während seiner Tätigkeit in Zürich versah er mehrmals Gastprofessuren im Ausland, so u.a. in Berkeley, Ann Arbor, Tokio, Pisa und Rom. Von 1987 bis 1990 war er Präsident der ETH Zürich und Vizepräsident des Schweizerischen Schulrates. Für seine wissenschaftliche Tätigkeit in der Versicherungsmathematik erhielt Hans Bühlmann mehrere Auszeichnungen, darunter vier Ehrendoktorate.

\section{Ein Experiment}

Wenn ich Ihnen die Frage stelle: "Was ist das?" (siehe Abb. 1), so werden Sie mit grosser Wahrscheinlichkeit antworten: "die ETH" oder vielleicht "das Hauptgebäude der ETH". Sie werden, obwohl Ihnen der gedankliche Unterschied grundsätzlich sehr wohl vertraut ist, nur selten die präzisere Antwort wählen: "das ist ein Bild der ETH" oder "das ist ein Bild des Hauptgebäudes der ETH". Natürlich reflektiert das Bild, so wie alle Bilder, den Gegenstand, den es abbildet, nur mangelhaft. Schon die Anzahl Fenster des Hauptgebäudes der ETH ist an dem gezeigten Bild nicht feststellbar, geschweige denn

Aussagen, die aus mathematischen Modellen gewonnen werden, sind nur so gut wie die Modelle selbst. Eine Binsenwahrheit! Sie impliziert, dass der Anwender über Inhalt und Grenzen der verwendeten Modelle genau orientiert sein muss und sie nur dort einsetzt, wo er sie für gülttig hält. Diese an sich selbstverständliche Forderung ist im Zeitalter von umfangreichen Computerprogrammen für komplizierte Aufgaben nur schwer zu erfüllen. Es bleibt der Mathematik trotzdem die Verpflichtung, die Voraussetzungen und die Wirkungsweisen der Modelle dem Anwender immer wieder darzulegen und zu erklären. Allerdings muss der Adressat die Erklärungen auch aufnehmen, und dies bedingt auf seiner Seite sicher auch eine hinreichende Vorbildung in Mathematik! Hans Bühlmann beschäftigt sich mit zwei mathematischen Paradigmen der Finanzwirtschaft, die sich aus verschiedenen Bedürfnissen heraus entwickelt haben. Im falschen Umfeld unkritisch angewendet können sie leicht zu unliebsamen Resultaten führen. Der Text ist eine leicht überarbeitete Fassung der Abschiedsvorlesung des Autors, die am 2. Juli 1997 an der ETH Zürich stattgefunden hat. ust 


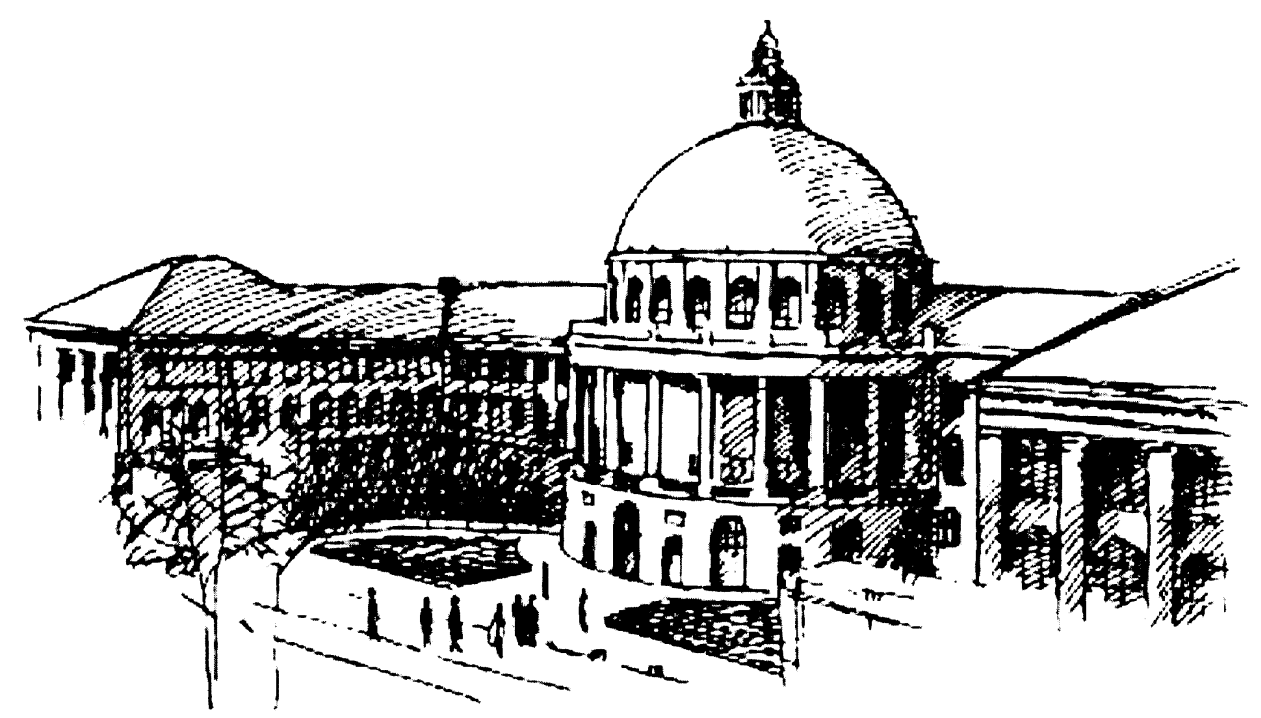

Abb. 1

die Materialbeschaffenheit oder die Farbe der Fassade, und schon gar nicht jegliche Information über das Innere des Gebäudes.

Was ich Ihnen mit diesem Experiment demonstrieren wollte, ist die Tatsache, dass wir Menschen in der Kommunikation, wie im Denken, Bilder mit den von ihnen dargestellten Gegenständen immer wieder identifizieren. Wenn wir genügend sorgfältig denken und uns genügend sorgfältig ausdrücken, sind wir uns des Unterschiedes von Bild und dargestelltem Objekt zwar sehr wohl bewusst; aber trotzdem ist die Identifikation von Bild und Gegenstand für uns oft eine der fruchtbarsten Möglichkeiten, um zu kommunizieren und zu denken. Im Bereich der Ideen ist es vor allem die Wissenschaft, welche solche Bilder liefert. Sie heissen dann Theorien oder eben Paradigmen.

\section{Mathematische Paradigmen in der Finanzwirtschaft}

Ich habe mir für heute vorgenommen, Ihnen die Grundmuster zweier Paradigmen nachzuzeichnen, welche die Mathematik im Bereich der Finanzwirtschaft geschaffen hat. Mathematische Paradigmen in der Finanzwirtschaft benützen vor allem die Sprache der Wahrscheinlichkeitstheorie. Diese ist ein relativ junger Zweig der Mathematik. Sie hat nichts mit den Babyloniern, Ägyptern, Griechen und meines Wissens auch nichts mit den Chinesen und Indern zu tun. Sie ist ein Kind des Abendlandes, zeitlich geboren in der Renaissance.

Die erste schriftliche Spur eines Problems, das klar der Wahrscheinlichkeitstheorie zuzuordnen ist, findet sich in der "Summa de arithmetica, geometria, proportioni et proportionalita", publiziert 1494 von Fra Luca Pacioli (siehe Abb. 2). Es handelt sich bei der "Summa" um das erste gedruckte Werk, welches den Versuch unternahm, das mathematische und rechnerische Wissen des ausgehenden 15. Jahrhunderts in enzyklopädischer Form darzustellen. Bemerkenswert an diesem Werk ist, dass es auch die erste historische 


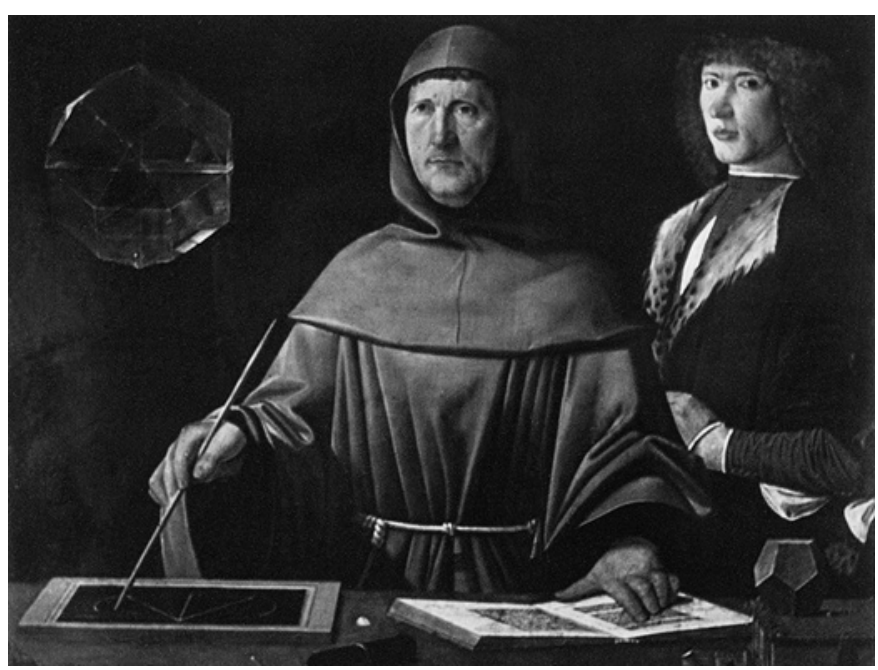

Abb. 2 Fra Luca Pacioli 1445-1514 (circa), Franziskanermönch, geboren in Borgo (Toscana); unterrichtete Mathematik in Perugia, Rom, Neapel, Pisa und Venedig, bevor er als Mathematikprofessor an den Hof Ludovico Sforzas nach Mailand kam. Dort lernte er Leonardo da Vinci kennen. Leonardo und Fra Luca verliessen beide Mailand, als Louis XII dort einzog. Seine letzten Jahre verbrachte Fra Luca in Florenz.

Belegung der doppelten Buchhaltung enthält, was Fra Luca Pacioli die Ehre eingetragen hat, als Vater des modernen Rechnungswesens gefeiert zu werden.

\section{Gioco di Balla}

Doch nun zum wahrscheinlichkeitstheoretischen Problem, das sich in der "Summa" befindet: Fra Luca nennt es Gioco di Balla, später heisst es auch Jeu des Pistolets (bei den Franzosen) und Problem of Points (bei den Briten).

Die Personen $A$ und $B$ spielen ein faires Spiel mehrere Male: Wer als erster 6 Siege errungen hat, soll die eingesetzte Summe erhalten. Nun brechen sie ab bei der Situation, wo $A 5$ Siege und $B 3$ Siege aufzuweisen hat. Wie soll die Summe aufgeteilt werden?

Die Antwort von Pacioli lautet:

Die Aufteilung soll im Verhältnis $5: 3$ erfolgen.

Aus der Sicht der Wahrscheinlichkeitsrechnung ist diese Antwort eindeutig falsch. Sie ist nur erklärbar aus der Nichterkenntnis des wahrscheinlichkeitstheoretischen Charakters der Problemstellung. Fra Luca war befangen im reinen Proportionalitätsdenken, was ja auch im Titel der Summa zum Ausdruck kommt.

Es dauerte 160 Jahre, bis das von Fra Luca publizierte Problem gelöst wurde. Dokumentiert ist die Lösung in einem berühmten Briefwechsel zwischen Fermat (siehe Abb. 3) und Pascal (siehe Abb. 4) im Jahre 1654. 


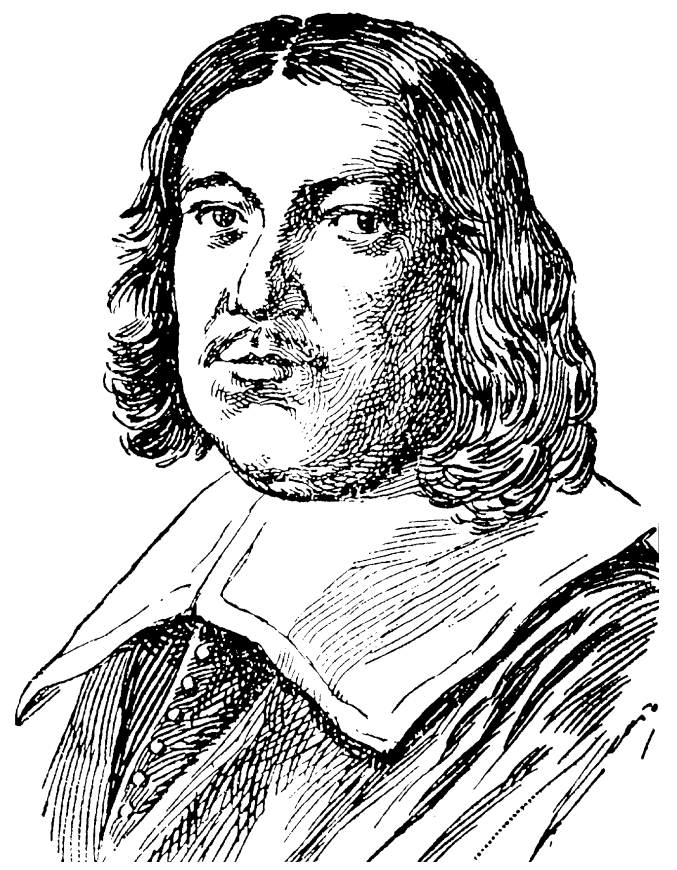

Abb. 3 Pierre de Fermat (1601-1665), geboren in Beaumont-de-Lomagne. Studierte Recht in Toulouse und Orléans und Mathematik in Bordeaux. Mit 30 Jahren wurde er Conseiller de la Chambre des Requêtes du Parlement in Toulouse. Er lebte seit diesem Zeitpunkt in Toulouse. Seine richterlichen Funktionen verboten ihm jede andere lukrative Tätigkeit. Deshalb widmete er sich als "Amateur" den Sprachen, der Poesie und der Mathematik. In der Mathematik sind seine bekanntesten Arbeiten der Algebra gewidmet. Er beschäftigte sich auch mit der Bestimmung des Gewichtes der Erde und - was uns hier interessiert - mit der Wahrscheinlichkeitstheorie.

Fermats Lösung. Nach spätestens 3 weiteren Spielen würde die ganze Summe einem der Spieler zugesprochen. Fermat erstellte dann die Liste der Möglichkeiten für die drei fehlenden Spiele. In moderner Sprechart würden wir sagen, dass Fermat ein gedankliches Modell konstruierte. Für die Darstellung verwenden wir das Symbol $a$, um auszudrücken, dass $A$ gewinnt.

\begin{tabular}{|c|c|c|}
\hline Gewinnfolge & Zusprach & e der Sumr \\
\hline$a a a$ & $A \quad)$ & \\
\hline$a a b$ & $A$ & \\
\hline$a b a$ & $A$ & \\
\hline$a b b$ & $A$ & 7 Fälle \\
\hline$b a a$ & $A$ & \\
\hline$b a b$ & $A$ & \\
\hline$b b a$ & $A$ & \\
\hline$b b b$ & $B\}$ & 1 Fall \\
\hline
\end{tabular}




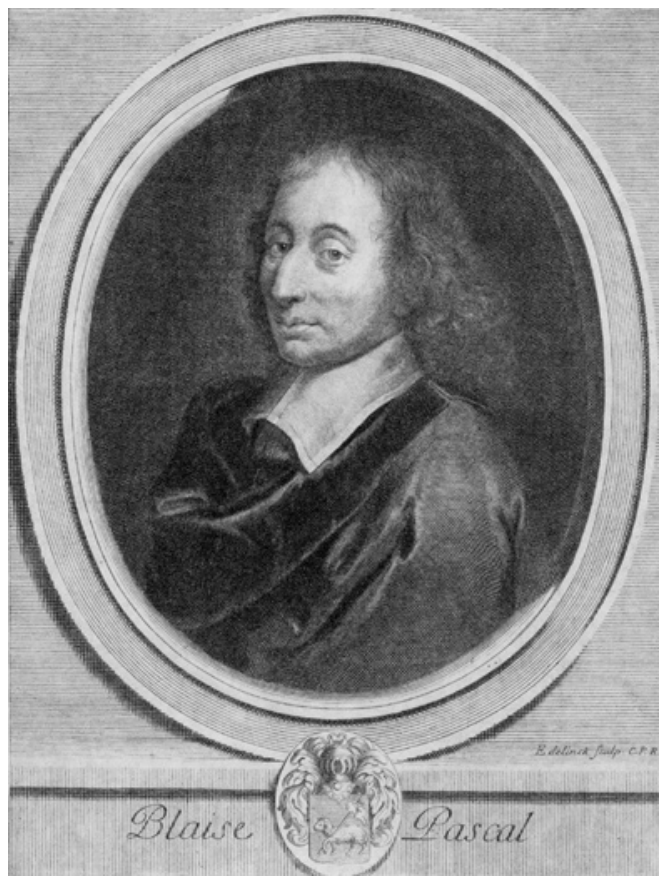

Abb. 4 Blaise Pascal (1623-1662), geboren in Clermont-Ferrand. Pascal war ein Wunderkind und wurde von seinem Vater schon mit 16 Jahren in den Gelehrtenkreis des Abbé Marin Mersenne in Paris (aus diesem Kreis entstand später die Académie Royale) eingeführt. Er war sein Leben lang hin- und hergerissen zwischen Mathematik und religiösem Eifer für die Werte des Jansenismus. In der Mathematik sind vor allem seine Beiträge zur projektiven Geometrie, Kombinatorik und Infinitesimalrechnung bekannt. Er war aber auch an physikalischen Problemstellungen (Vakuum, Kosmologie) sehr interessiert; zudem konstruierte er eine funktionsfähige Rechenmaschine.

Unter der Annahme, dass alle Gewinnfolgen gleiche Wahrscheinlichkeiten haben, ergibt sich als Fermats Lösung:

Die Aufteilung soll im Verhältnis $7: 1$ erfolgen.

Diese Lösung teilte Fermat in einem Brief an Blaise Pascal mit.

Pascals Lösung. Pascal löste das Problem mit einer komplett verschiedenen Methode, welche wir heute als rekursiv bezeichnen würden. Symbolisch lässt sich die Methode am besten anhand einer Baumstruktur nachvollziehen (siehe Abb. 5). Wir gehen von der Gewinnsituation $5: 3$, also 5 Siege für $A$ und 3 Siege für $B$ im Moment des Abbruchs aus und zeichnen auf, wie die Gewinnsituation sich entwickeln könnte.

In den Endpunkten links erhält $A$ die ganze Summe. Im Endpunkt rechts erhält $B$ die ganze Summe. Nehmen wir an, die ganze Summe sei 8 und zeichnen wir den Gewinn für $A$ in den Endpunkten ein. Der Gewinn für $B$ ist an jeder Stelle das Komplement zu 8. Gehen wir nun rückwärts durch den Baum durch "Mittelung der möglichen Gewinne", 
Annahme: Summe $=8$

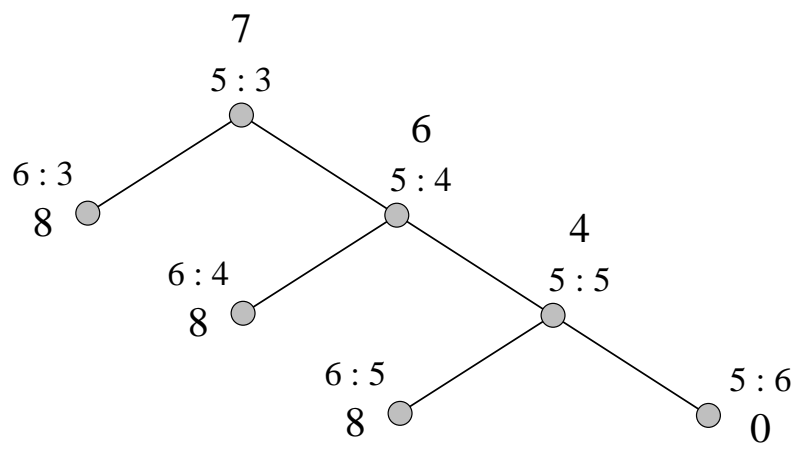

Abb. 5 Pascalsches Schema.

so erhalten wir

$$
\begin{array}{ll}
\text { bei } 5: 5 & \mathbf{4}=\frac{8+0}{2} \\
\text { bei } 5: 4 & \mathbf{6}=\frac{8+4}{2} \\
\text { bei } 5: 3 & \mathbf{7}=\frac{8+6}{2}
\end{array}
$$

Damit fand auch Pascal, dass die Summe im Verhältnis

$$
7(\text { Gewinn von } A) \quad: \quad 1(\text { Gewinn von } B)
$$

aufzuteilen sei.

Der Brief, in welchem Pascal seine Lösung an Fermat mitteilte, ist erhalten geblieben. Er trägt das Datum vom 29. Juli 1654 und enthält den berühmten freudvollen Satz: "Je vois bien que la vérité est la même à Toulouse et à Paris". Die Wahrscheinlichkeitstheorie war geboren!

\section{Die drei Grundideen, welche der Wahrscheinlichkeitstheorie zugrunde liegen}

In der Korrespondenz zwischen Fermat und Pascal sind die drei Grundideen der Wahrscheinlichkeitstheorie sichtbar, die auch heute noch einen wesentlichen Teil des Fundaments dieser mathematischen Disziplin darstellen:

- Fermats Liste: Sie ist das, was wir heute das Modell möglicher Szenarien nennen würden. Mathematisch wird sie dargestellt durch den Wahrscheinlichkeitsraum mit seinen möglichen Elementar- und zusammengesetzten Ereignissen.

- Pascals rekursive Methode: Die geniale Idee Pascals bestand in seiner Entdeckung, dass man statt der vollen Aufzählung aller möglichen Szenarien (globales Modell) 
auch oft nur lokal richtig evaluieren muss, um die im globalen Modell definierten Wahrscheinlichkeiten zu finden. Der Vorteil dieser Methode ist vor allem ihre Praktikabilität, weshalb man sie z.B. in der heutigen Optimierungstheorie immer wieder antrifft (Backward Induction, Roll Back Analysis).

- Fairness des Spiels: In allen unseren Überlegungen haben wir unterstellt, dass alle Möglichkeiten gleiches Gewicht oder eben gleiche Wahrscheinlichkeit haben. In einem Fall waren es die Elemente von Fermats Liste, im anderen die alternativen Verzweigungsäste im Pascalschen Schema. In der modernen Wahrscheinlichkeitstheorie ist aus diesem Grundgedanken die Martingaltheorie entstanden, welche in Theorie und Praxis eine bedeutende Rolle spielt.

Lassen Sie mich nun illustrieren, wie diese Grundideen der Wahrscheinlichkeitstheorie in die zwei mathematischen Paradigmen der Finanzwirtschaft einfliessen, welche insbesondere die bedeutenden Zweige der Versicherung und der Derivativen Finanzinstrumente prägen.

\section{Paradigma der Versicherungsmathematik: Gambler's Ruin}

Die wahrscheinlichkeitstheoretische Bemessung des Ruins im Rahmen der Glücksspiele gehört historisch zu den ältesten Problemen der Wahrscheinlichkeitstheorie. Ich verwende für die Bezeichnung dieses Problems den weltweit üblichen englischen Namen: "Gambler's Ruin". Es taucht erstmals auf als "Fünftes Problem" in dem Buch "De Ratiocinis in Ludo Aleae" (erschienen 1657) von Christiaan Huygens (siehe Abb. 6). Gestatten Sie mir die Problemstellung in heutiger Sprechweise wiederzugeben:

"Ich spiele mit eigenem Vermögen $a$ gegen einen Gegner mit Vermögen $b$, bis einer von uns beiden ruiniert ist. Wie gross ist die Wahrscheinlichkeit, dass ich ruiniert werde?"

Wir bezeichnen in der Folge die Wahrscheinlichkeit für meinen Ruin mit $P[$ Ruin]. Dem Problem sei eine (beliebig fortsetzbare) Folge von fairen Spielpartien mit Einsatz 1 zugrunde gelegt, was die graphische Darstellung in Abb. 7 zum Ausdruck bringt.

Falls der Austritt unten stattfindet, bin ich ruiniert, bei Austritt oben ist mein Gegner ruiniert. Zur gedanklichen Lösung von "Gambler's Ruin" wäre es nötig, eine Liste mit sämtlichen möglichen Kurven $\left(S_{n}\right)_{n \geq 0}$ (Szenarien) zu erstellen. Wie Sie leicht sehen, gibt es allerdings eine unendliche Anzahl solcher Kurven, denn die Spieldauer kann ja beliebig gross sein. Damit ist das "Abzählen der Szenarien" im elementaren Sinne nicht mehr möglich. Die Mathematiker haben aber die notwendigen Instrumentarien entwickelt, um auch in solchen Situationen Wahrscheinlichkeitsräume und Wahrscheinlichkeiten exakt zu definieren. Es würde allerdings weit über den Rahmen dieses Vortrages hinausgehen, Ihnen dies heute erklären zu wollen. Umso erstaunlicher ist dafür die Tatsache, dass sich auch ohne dieses Verständnis meine Ruinwahrscheinlichkeit berechnen lässt.

Dazu benötigen wir lediglich die Fairnessbedingung, die besagt, dass mein erwarteter Gewinn in jedem Zeitpunkt gleich gross ist wie mein Gewinn zu Beginn des Spieles, 


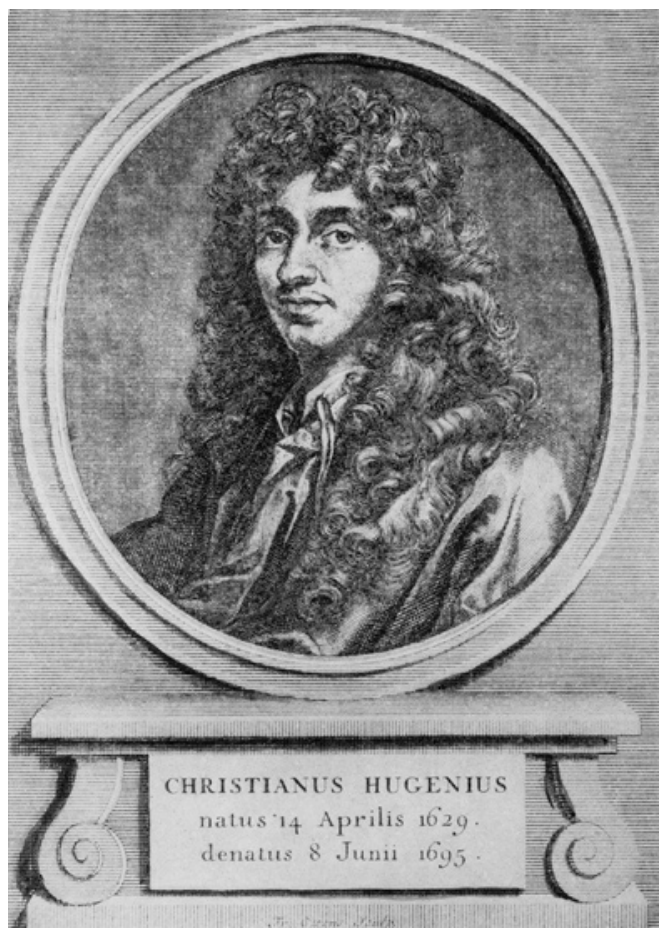

Abb. 6 Christiaan Huygens (1629-1695). Christiaan Huygens wurde in Den Haag geboren. Zuerst von seinem Vater in Mathematik und klassischen Sprachen unterrichtet, studierte er dann in Leiden und Breda Recht und Mathematik. Er besuchte öfters Paris und London und war Mitglied der Royal Society wie der Académie Royale des Sciences. Der erste Besuch Huygens in Paris fand im Jahre 1655 statt. Er vernahm dabei von der Korrespondenz zwischen Fermat und Pascal, traf aber mit keinem der beiden zusammen. Da ihm zudem niemand die Ansätze von Fermat und Pascal erklären konnte, begann er nach seiner Rückkehr nach Holland selbständig mit der Bearbeitung der Lösungen der ihm mitgeteilten Probleme. Daraus entstand 1656 ein holländisches Manuskript und 1657 "De Ratiocinis". Dieses letztere Werk kann man als das historisch erste Textbuch der Wahrscheinlichkeitstheorie bezeichnen. Erst zu Beginn des 18. Jahrhundert entstand die nächste Generation von Textbüchern (Montmort, de Moivre, Jakob Bernoulli).

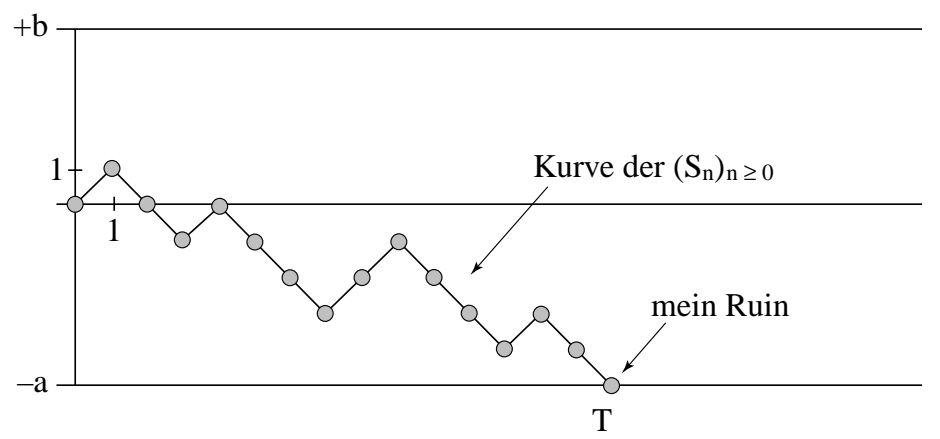

Abb. 7 Irrfahrt der aufsummierten Gewinne. Folge von fairen Spielen mit +1 und -1 als mögliche Resultate. Es bezeichnet $S_{n}$ meinen aufsummierten Gewinn nach $n$ Spielen, und $T$ bedeutet die Zeit des Austritts aus $(-a, b)$ (stochastische Zeit). 
also Null; es gilt also für alle $n$ :

$$
\begin{array}{cc}
E\left[S_{n}\right]=\sum_{k=-n}^{+n} k \cdot P\left[S_{n}=k\right]=0 . \\
\begin{array}{c}
\uparrow \\
\text { Definition } \\
\text { des } \\
\text { Erwartungswertes }
\end{array} & \begin{array}{r}
\text { Fairness- } \\
\text { bedingung }
\end{array}
\end{array}
$$

Die Fairnessbedingung gilt auch zur stochastischen Zeit $T$. (Das scheint intuitiv einleuchtend, ist aber im Kern die Aussage eines sehr tiefliegenden mathematischen Satzes (Stoppsatz der Martingaltheorie), dessen Richtigkeit unter sehr allgemeinen Bedingungen bewiesen werden kann.) Es gilt also

$$
E\left[S_{T}\right]=0
$$

oder, anders ausgedrückt

$$
-a \cdot P[\text { mein Ruin }]+b \cdot P[\text { Gegners Ruin }]=0 .
$$

Zusätzlich gilt

$$
P[\text { mein Ruin }]+P[\text { Gegners Ruin }]=1 .
$$

Die letzte Gleichung bedeutet, dass bei beliebig langer Spieldauer mit Sicherheit einer von beiden ruiniert sein wird. Aus den zwei letzten Gleichungen folgt unmittelbar

$$
P[\text { mein Ruin }]=\frac{b}{a+b}
$$

Eine direkte Konsequenz aus dieser Formel ist die Tatsache, dass $P$ [mein Ruin] gegen 1 strebt, wenn $b$ gegen $\infty$ strebt, d.h. wenn $b$ sehr gross wird. In Worten heisst dies:

Ein faires Spiel gegen einen sehr reichen Gegner kann man auf die Dauer nicht durchhalten; man verliert gegen ihn mit Sicherheit jeden Einsatz.

Konkrete Beispiele für diese Situation sind die folgenden Fälle:

- Individueller Spieler gegen Spielkasino (hier hat zudem das Spielkasino noch den Vorteil, dass das Spiel zu seinen Gunsten unfair ist)

und

- Versicherungsgesellschaft gegen Versicherungsmarkt.

Dieses letzte Beispiel unterstellt, dass der gesamte Versicherungsmarkt gegenüber der einzelnen Versicherungsgesellschaft "sehr reich" ist. In dieser Situation ergibt sich die 


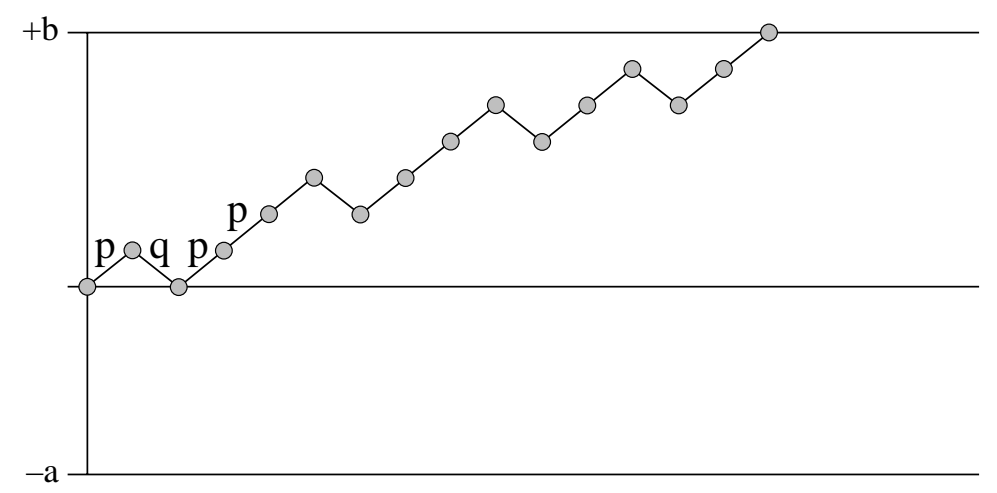

Abb. 8 Irrfahrt der aufsummierten Gewinne.

praktische Konsequenz, dass die Versicherungsprämien zur fairen Prämie einen Sicherheitszuschlag benötigen. Auch dieser Fall lässt sich mathematisch in den Griff kriegen. Studieren wir zu diesem Zweck nochmals die Irrfahrt der aufsummierten Gewinne, d.h. die Kurven $\left(S_{n}\right)_{n \geq 0}$ (siehe Abb. 8).

Der einzige Unterschied zur früheren Situation ist der, dass der Schritt +1 mit Wahrscheinlichkeit $p$ erfolgt und der Schritt -1 mit Wahrscheinlichkeit $q=1-p$. Dabei gelte $p>q$. Die Fairnessbedingung $p=1 / 2$ ist damit fallengelassen. Wir haben jetzt

$$
\frac{p}{q}=1+\Theta,
$$

wobei $\Theta$ der Sicherheitszuschlag ist. Auch in diesem Fall der (nichtsymmetrischen) Irrfahrt ist die Formel für die Ruinwahrscheinlichkeit schon lange bekannt. Sie steht als Übungsaufgabe - in der 1713 publizierten "Ars Conjectandi” von Jakob Bernoulli (siehe Abb. 9):

$$
P[\text { mein Ruin }]=\left(\frac{q}{p}\right)^{a} \frac{1-\left(\frac{q}{p}\right)^{b}}{1-\left(\frac{q}{p}\right)^{a+b}} .
$$

Basierend auf dem Gambler's Ruin Paradigma im Falle der nichtsymmetrischen Irrfahrt hat der Schwede Filip Lundberg (siehe Abb. 10) im Jahre 1903 die Kollektive Risikotheorie für das Versicherungsgeschehen entwickelt. Sie setzt sich zum Ziel, dieses Versicherungsgeschehen als eine zufällige Entwicklung in der Zeit zu modellieren. Die Kontrolle über das Versicherungsgeschehen wird dabei mittels der Ruinwahrscheinlichkeit gesteuert, d.h. man definiert als zulässige Versicherungsoperationen solche, die ein vorgegebenes Niveau für die Ruinwahrscheinlichkeit nicht überschreiten.

Die Bernoullische Formel liefert uns bereits den Schlüssel zur Kontrolle mittels der Ruinwahrscheinlichkeit. Etwas anders geschrieben (in Analogie zur Notation in der Risikotheorie) lautet sie:

$$
P[\text { Ruin }]=e^{-R a} \cdot C_{a b} .
$$




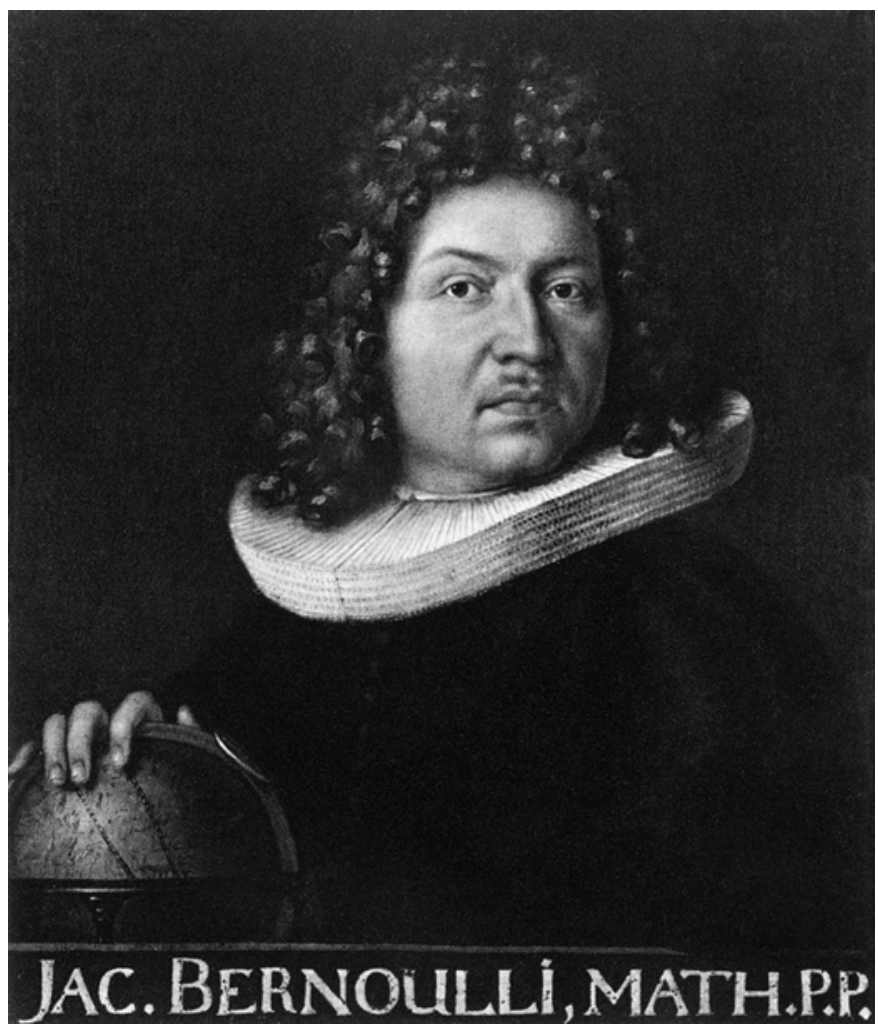

Abb. 9 Jakob Bernoulli (1654-1705). Die Familie Bernoulli kam anfangs des 16. Jahrhunderts aus Antwerpen über Frankfurt am Main nach Basel. Niklaus Bernoulli war während einiger Jahre Bürgermeister der Stadt Basel. Sein ältester Sohn Jakob studierte zunächst Theologie in Basel, interessierte sich aber immer mehr für Mathematik. In dieser Disziplin entstand zwischen ihm und seinem jüngeren Bruder Johann eine eigentliche Familienfehde, wer der bessere Mathematiker sei. Dies erklärt auch die erst postume Veröffentlichung der "Ars Conjectandi" (1713). Dieses Werk fasst viele der bisherigen Ergebnisse der Wahrscheinlichkeitstheorie zusammen und erweitert die Problemstellungen. Mit dem "Gesetz der grossen Zahlen" wird in der "Ars Conjectandi" erstmals die theoretische Brücke zwischen Wahrscheinlichkeitstheorie und Statistik geschlagen.

Dabei gilt $0 \leq C_{a b} \leq 1$ und $C_{a b} \rightarrow 1$ für $b \rightarrow \infty$, sowie $R=\ln (1+\Theta)$. Das Versicherungsgeschehen wird also durch die Parameter $a$ und $R$ kontrolliert, wo $a$ die Kapitalisierung bezeichnet und $R$ durch den Sicherheitszuschlag $\Theta$ und durch die Portefeuillestruktur kontrolliert wird. Die Portefeuillestruktur geht in die Bernoullische Formel über den Kurventypus der Irrfahrt ein. Unterstellt man dem Risikoprozess andere Kurventypen, verändert sich der Wert von $R$ (siehe Abb. 11, 12).

Die kollektive Risikotheorie verbindet also Kapitalisierung, Tarifierung und Risikopolitik eines Versicherungsunternehmens und leitet daraus einen Sicherheitsindex, eben die Ruinwahrscheinlichkeit ab. Der Versicherungspraktiker erkennt an dieser Stelle unmittelbar, dass damit ein theoretisches Instrumentarium zur Verfügung steht, um unter Einhaltung eines vorgegebenen Sicherheitsniveaus strategische Unternehmensentscheide 


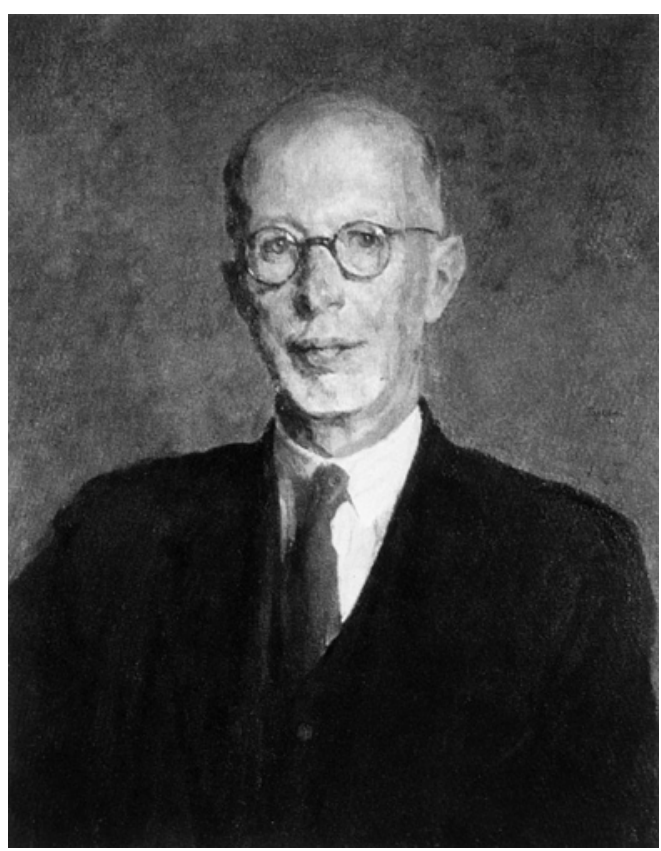

Abb. 10 Filip Lundberg (1876-1965). Geboren in Stockholm, studierte Mathematik und schrieb seine Dissertation in Stockholm. In dieser Dissertation wurde erstmals das Versicherungsgeschehen als stochastischer Prozess interpretiert. Dies, obwohl das mathematische Instrumentarium zur Behandlung stochastischer Prozesse noch nicht geschaffen war. Es blieb Harald Cramer vorbehalten, die Lundbergschen Ideen im streng mathematischen Sinn zu klären.

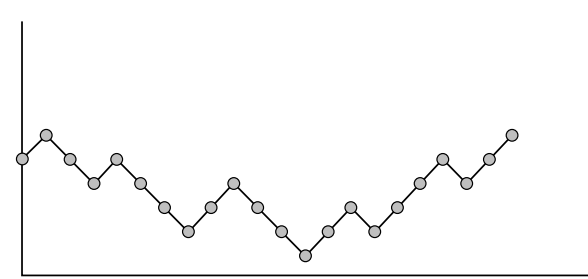

Abb. 11 Kurventyp: Irrfahrt.

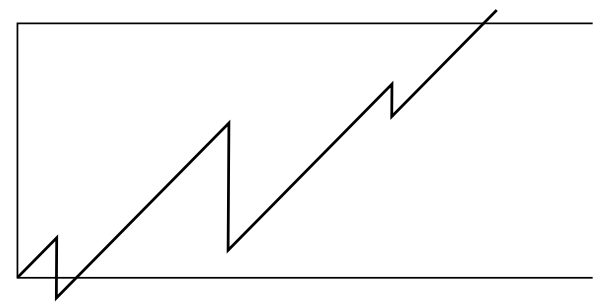

Abb. 12 Kurventyp: Verlauf Überschuss Prämien minus Schäden

betreffend Solvabilitätsmarge, Risikokapital, Tarifierungspolitik und Selbstbehaltspolitik aufeinander abzustimmen.

Grundhaltung, die aus dem Paradigma Gambler's Ruin resultiert. Das Gambler's Ruin Paradigma ist selbstverständlich ein mathematisches Denkschema, welches das Versicherungsgeschehen nur unvollkommen reflektiert. Die Tatsache, dass das Denkschema gewisse Grundhaltungen in der Versicherungsbranche geprägt hat, ist aber evident. Es sind dies: 
- Die langfristige Betrachtungsweise, d.h. die Modellierung des Risikoverlaufes für typischerweise langjährige Versicherungsbeziehungen steht im Vordergrund.

- Die Kontrolle des Risikos findet über die Anfangsbedingungen des Risikoprozesses statt. Nachher überlässt man das Risiko weitgehend den Gesetzen der Wahrscheinlichkeitstheorie.

Diese Haltung ist vor allem in der Lebensversicherungsbranche besonders deutlich erkennbar. Andere Versicherungsbranchen weichen gelegentlich durch zusätzliche Kontrollmechanismen, z.B. mittels variabler Prämien, Bonus/Malus, von dieser Langfristigkeit etwas ab.

\section{Paradigma der Finanzmathematik}

\section{(Bewertung von derivativen Finanzinstrumenten, Hedging)}

Die Grundidee des Hedging findet sich ebenfalls im Werk von Christiaan Huygens "De Ratiocinis in Ludo Aleae". Ich erkläre sie aber direkt im modernen Zusammenhang der Optionsbewertung. Mit ihrer Arbeit "The Pricing of Options and Corporate Liabilities", Journal of Political Economy (1973), haben Fischer Black (siehe Abb. 13) und Myron

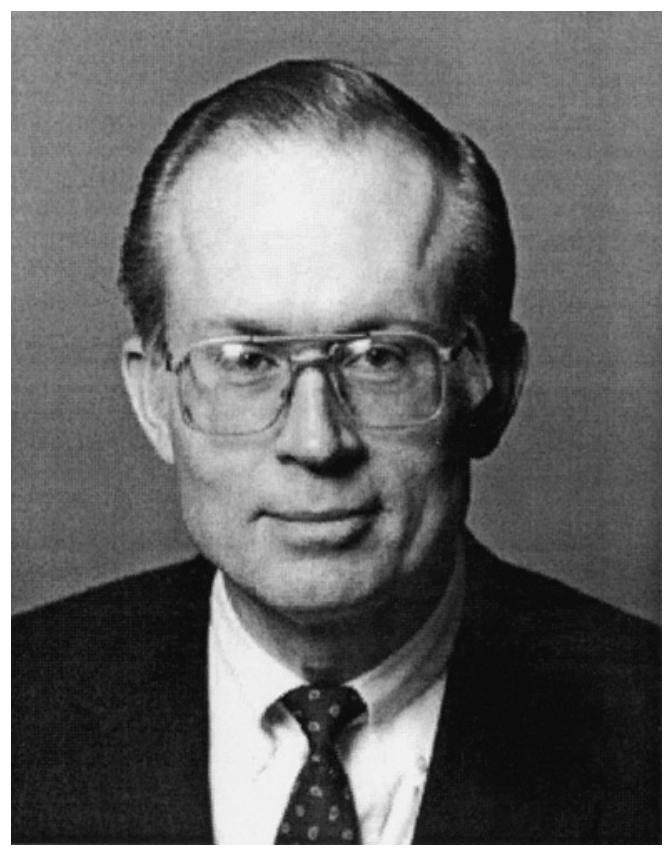

Abb. 13 Fischer Black (1938-1995). Studierte Physik und doktorierte in mathematischer Logik an der Harvard University. Als beweglicher Geist wechselte er dann in eine Unternehmensberaterfirma, wo sein Interesse für Finanzökonomie geweckt wurde. Er stieg im Umfeld der University of Chicago wieder in den Kreis der akademischer Ökonomen ein, wechselte dann an das MIT, um schliesslich bei Goldman Sachs letztlich doch wieder seine Laufbahn an der Front der Finanzpraxis einmünden zu lassen. Auch in dieser Stellung publizierte er aber laufend seine Ideen und Anregungen in den führenden ökonomischen Zeitschriften. Bei der Verleihung des Nobelpreises an Robert Merton und Myron Scholes (siehe Abb. 14) wurde er offiziell postum in die Ehrung eingeschlossen. 


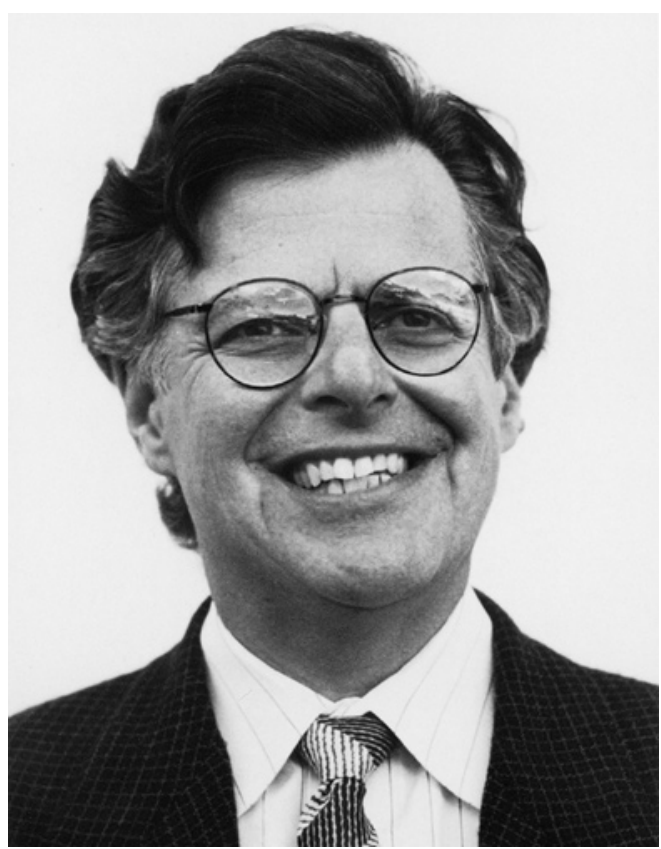

Abb. 14 Myron S. Scholes Geboren 1941, Professor der Stanford University, Nobelpreis für Ökonomie 1997.

Scholes (siehe Abb. 14) die eigentliche Grundlage für den Optionenmarkt und damit für die Bewertung von derivativen Finanzinstrumenten schlechthin geschaffen.

Stellen wir uns also die Aufgabe, folgenden Option zu bewerten:

Call Option europäisch

Ausübungszeitpunkt $T$

Ausübungspreis 1000

Die Option sei bezogen auf einen Basiswert (z.B. Aktie), der im heutigen (Bewertungs-) Zeitpunkt ebenfalls den Wert 1000 hat.

Wenden wir zunächst einmal etwas mechanistisch das Schema von Pascal an (siehe Abb. 15). Im Ausübungszeitpunkt sind die Werte 30, 10, 0, 0 die durch das Optionsrecht resultierenden Werte. Die Werte in den früheren Zeitpunkten erhalten wir durch Backward Induction.

Das Pascal-Schema liefert 7.5 als den heute zu verlangenden Preis. Wie lässt sich dieser Preis also rechtfertigen? Für diese Rechtfertigung verwenden wir das Hedging Argument (siehe Abb. 16).

Beachte, dass im Schema (siehe Abb. 16) die Bewertungsdifferenzen 10, 7.5, 5, 2.5 dem Betrage nach an jedem Knoten die gleichen sind und somit die Optionsbewertung die Bewertungsdifferenzen des Basiswertes proportional nachvollzieht. Allerdings ist der Proportionalitätsfaktor je nach Verlauf des Basiswertes verschieden. 


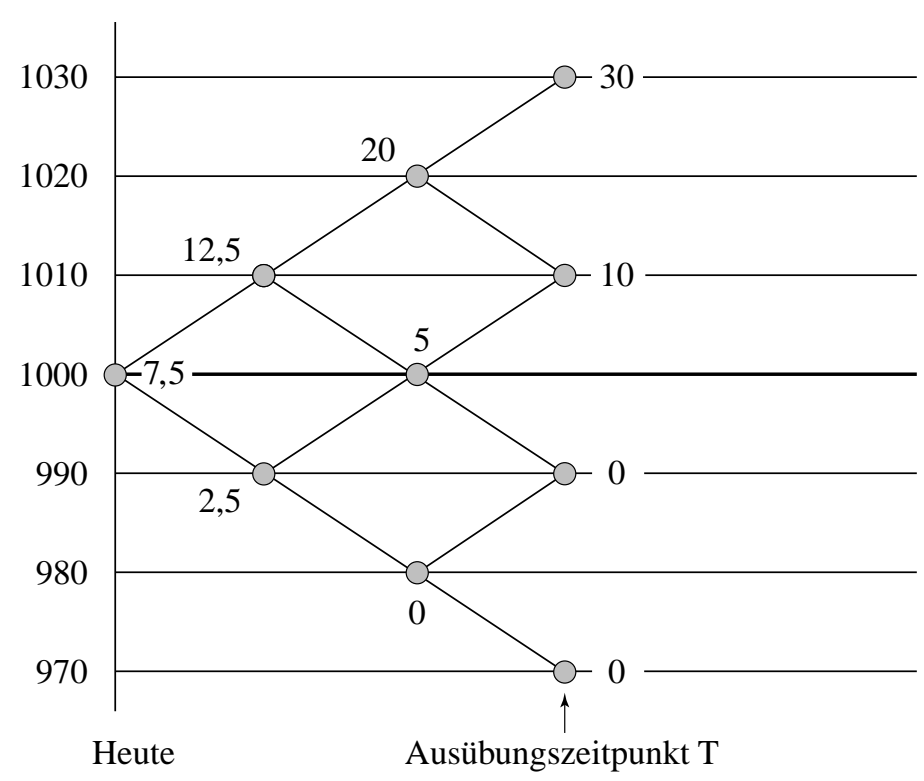

Abb. 15 Das Schema von Pascal.

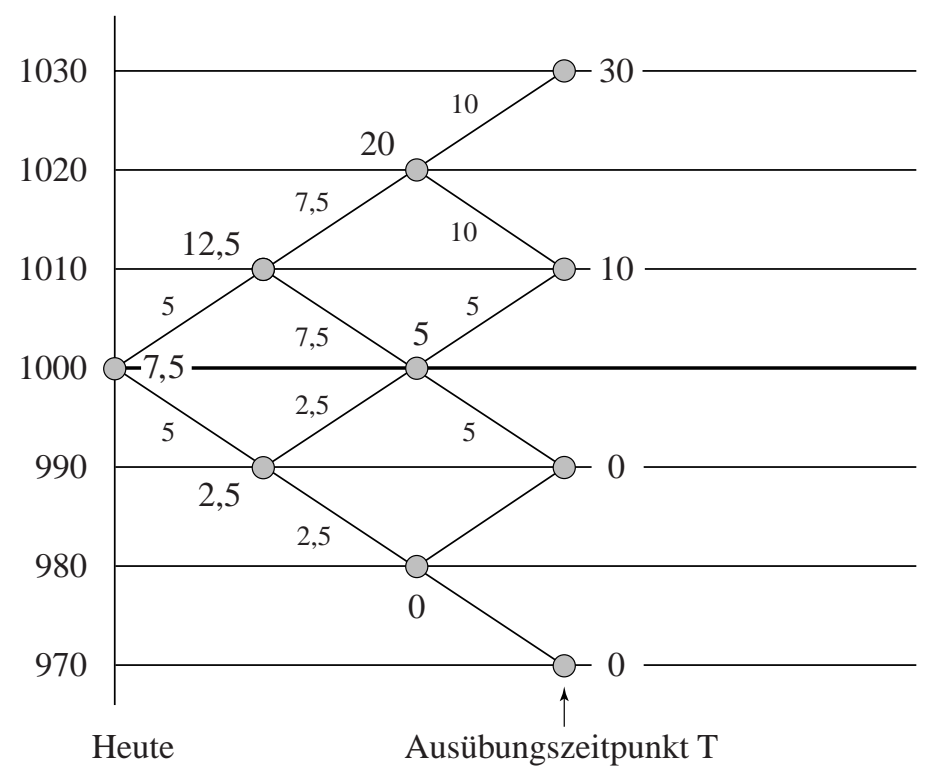

Abb. 16 Hedging Argument. 


$\begin{array}{cccc}\text { Bewertungsdifferenz } & 10 & 1 & \text { Aktie } \\ & 7.5 & 3 / 4 & \text { Aktie } \\ & 5 & 1 / 2 & \text { Aktie } \\ & 2.5 & 1 / 4 & \text { Aktie }\end{array}$

Wir ziehen daraus das folgende Fazit: Wenn ich mit 7.5 starte (Preis, den ich als Emittent der Option erhalte), in jedem Knoten die (gebrochene) Anzahl Aktien kaufe, welche gemäss obiger Tabelle der Bewertungsdifferenz entspricht, und diesen Kauf im nächsten Knoten glattstelle, dann habe ich mit Sicherheit im Ausübungszeitpunkt $T$ den Geldbetrag, der dem Optionsrecht entspricht.

Wer dieses Argument zum ersten Mal hört, ist perplex. Unabhängig vom Verlauf des Basiswertes ist am Schluss der exakt benötigte Geldbetrag vorhanden!

Und eine weitere Feststellung ist fast noch verblüffender:

Nehmen wir an, das Gitter unserer Irrfahrt sei so, dass wir mit irgend einer von 1/2 verschiedenen Wahrscheinlichkeit nach oben gehen. Das eben durchexerzierte Hedging Argument ändert sich deswegen überhaupt nicht. Also spielt es gar keine Rolle, mit welcher Wahrscheinlichkeit $p$ der Basiswert steigt (bzw. mit welcher Wahrscheinlichkeit $1-p$ er fällt). Das Pascal-Schema, welches der Preisbewegung die Fairness unterstellt (d.h. $p=1 / 2$ ), liefert auch in diesem Falle die richtige Antwort!

Haben wir das perfekte Paradigma gefunden? Fast scheint es so, denn

- wir haben kein Risiko.

- wir müssen nicht einmal die Wahrscheinlichkeit $p$ kennen. Die "künstliche" Wahrscheinlichkeit $p^{*}=1 / 2$, welche den Preisprozess zum fairen Spiel macht, liefert immer die richtige Antwort.

Der eben beschriebene Sachverhalt lässt sich auf recht allgemeine Preisprozesse (die Klasse der Diffusionsprozesse) verallgemeinern. Ebenso wichtig ist es aber, auf die Grenzen der angestellten Überlegungen hinzuweisen. Schon für eine Irrfahrt, bei welcher Sie in jedem Knoten drei Möglichkeiten haben, versagt das Hedging Argument!

Grundhaltung, die sich aus dem Hedging Paradigma ergibt. Selbstverständlich wissen Theoretiker und Praktiker um die Grenzen des Hedging Arguments, und je besser sie ihr Metier beherrschen, umso geschickter können sie mit diesen Grenzen umgehen. Trotzdem wird auch durch dieses Paradigma eine Grundhaltung geprägt.

- Die kurzfristige Sicht steht im Vordergrund, geht es doch in erster Linie darum, das Hedging ständig neu anzupassen.

- Der Zufallsprozess wird weniger durch die Anfangsbedingungen als durch dieses ständige Anpassen kontrolliert.

- Die Preise sind die dominanten Grössen, in dem Sinne, als oft nach der Maxime gehandelt wird, man hätte lediglich dafür zu sorgen, dass die Preise stimmen.

Die Problematik der Grundhaltung wird in der Praxis insbesondere im Bereich des Risikomananagements sichtbar. Wie ist das Risiko zu kontrollieren, welches im zugrunde 
liegenden Paradigma gar nicht vorhanden ist? So ist es denn gerade diese Frage, welche die Praktiker zunehmend auch im Finanzbereich eher mit Ansätzen aus der Versicherungswelt angehen.

\section{Das Zusammentreffen der zwei Paradigmen}

In der modernen Finanzwelt wachsen Bankenwelt und Versicherungswelt immer mehr zusammen. Damit treffen sich aber auch zwei verschiedene Denkweisen, wie ich Ihnen das in Abb. 17 nochmals zusammengestellt habe. Dieses Aufeinandertreffen beinhaltet auch rein gedanklich viel Zündstoff. Letztlich kann aus dem Treffen der beiden Paradigmen aber auch eine fruchtbare Interaktion entstehen. Dieser Prozess läuft gegenwärtig vor allem im Bereich der Versicherungsderivate (Versicherungsdeckungen in Form von Finanzprodukten). Diese hatten einen sehr mühsamen Start, weil sie eben nur dann sinnvoll sein können, wenn beide Seiten mit ihren verschiedenen Denkweisen ihre Expertise voll einbringen.

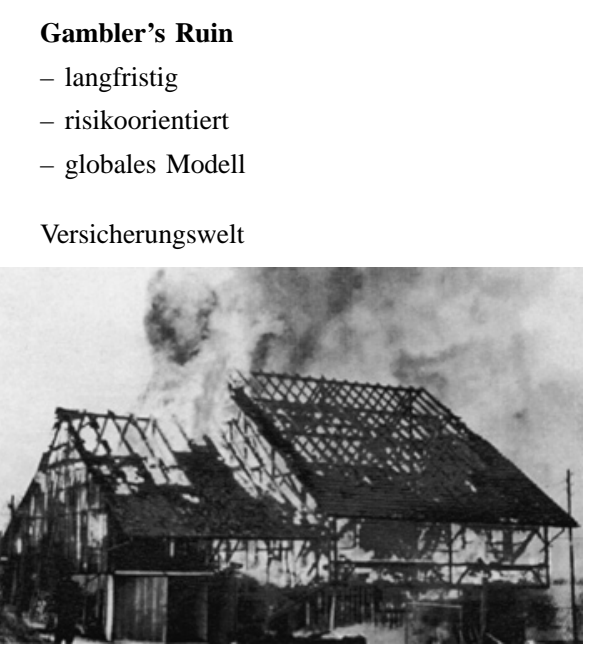

Abb. 17 Das Zusammentreffen der zwei Paradigmen.

\section{Hedging}

- kurzfristig

- preisorientiert

- lokale Lösung

Derivative Welt

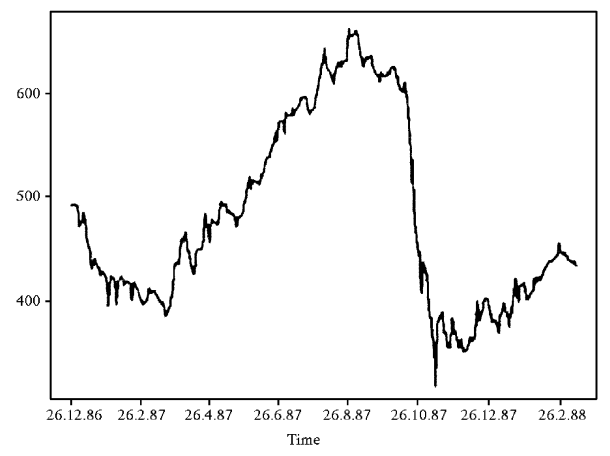

Gerade jetzt tauchen aber die ersten Beispiele auf, die diesem Postulat zu genügen vermögen.

Ja, jetzt sollte ich Ihnen wohl nochmals das Bild des Hauptgebäudes der ETH zeigen. $\mathrm{Zu}$ Beginn meiner Vorlesung habe ich damit auf den Unterschied zwischen Bild und abgebildetem Gegenstand hingewiesen.

Und so ist es natürlich noch umso mehr mit den zwei Paradigmen, die ich Ihnen vorgeführt habe:

- Gambler's Ruin ist nicht die Versicherungswelt.

- Hedging ist nicht die Derivative Welt. 
Aber so absolut ist das auch nicht. Gerade die beiden geschilderten Paradigmen haben das Denken in ihren Bereichen verändert. Ja, es ist meine persönliche Feststellung, dass in der Finanzwelt die Vorstellungen, welche die Verantwortlichen haben, meist entscheidender sind als die zugrundeliegenden ökonomischen Fakten. Mathematische Methoden nehmen bei diesen Vorstellungen oft eine besondere Stellung ein. In einer typischen SchwarzWeiss-Haltung werden sie in der Praxis oft entweder gar nicht zur Kenntnis genommen oder dann in fast bedingungsloser Gläubigkeit akzeptiert. Damit werden die Paradigmen von Bildern der Welt zu Machern der Welt.

Mir persönlich kommen unsere mathematischen Paradigmen deshalb gelegentlich wie eine Art Zauberlehrlinge vor, die wir spielerisch in die Welt gesetzt und die sich dann verselbständigt haben. Viele von uns haben auch schon praktische Erfahrungen gemacht, bei denen sie am liebsten gerufen hätten: "In die Ecke, Besen, der Du gewesen!" Aber leider geht das nicht, und es wäre auch nicht sinnvoll. Wir haben eine Mitverantwortung zu tragen, und die besteht darin, unsere Ansätze ständig und immer wieder so zu kommunizieren, dass sie wirklich verstanden werden. Ein wirklich guter Mathematiker möchte Ihnen, meine Damen und Herren, seine Formeln (moderner: seine Computerprogramme) nicht einfach verkaufen. Er ist viel mehr daran interessiert, dass Sie seine Überlegungen verstehen. Wenn das gelingt, sind die Zauberlehrlinge gezähmt, und dann können sie auch hervorragende Dienste leisten.

Hans Bühlmann

Mathematik

ETH-Zentrum

CH-8092 Zürich 